\title{
EFFECTIVENESS OF NATIONAL AIRLINES IN EUROPE - THE DEA APPROACH
}

Agata Żółtaszek, Ph.D.

University of Lodz

Faculty of Economics and Sociology

Institute of Spatial Economics

Department of Spatial Econometrics

37 Rewolucji 1905 r. Street, 90-214 Lódź, Poland

e-mail: zoltaszek@uni.lodz.pl

Renata Pisarek, Ph.D.

University of Lodz

Faculty of Economics and Sociology

Institute of Applied Economics and Informatics

Department of Logistics

37 Rewolucji 1905 r. Street, 90-214 Łódź, Poland

e-mail: renata.pisarek@uni.lodz.pl

Received 16 January 2016, Accepted 6 October 2016

\begin{abstract}
National airlines operate in a highly competitive environment. EU airlines face a challenge to compete with low cost carriers, as a result of the liberalization process in the sector. European flag airlines of nonEU member states, not benefiting from liberalization, are forced to compete internationally. This research is focused on national carriers, as they provide the majority of service to and from central and regional airports. Therefore, to establish the most efficient entities on the passenger air transport market, DEA (Data Envelopment Analysis) methodology, has been utilized. The purpose of this paper is to evaluate the effectiveness of 29 chosen national airlines in Europe in the year 2013, using the DEA approach, to pinpoint the subset of fully-efficient market leaders, as well as potential sources of inefficiency, among less effective carriers. The analysis incorporates information on inputs (e.g. fleet, number of employees, number of countries and airports served) and outputs (revenue, annual passengers carried, load factor). The results show that more than $40 \%$ (12 of 29) researched airlines are effective and the other $34 \%$ are near-efficient. Moreover, outcomes suggest that "going big" may not increase effectiveness. It is harder to achieve full efficiency for big carriers than small ones.
\end{abstract}

Keywords: Air transport, national airlines, transport economics, transport efficiency, Data Envelopment Analysis (DEA)

JEL classification: L93, C14, C44 


\section{Introduction}

Air transport is the fastest mode of transportation and is considered to be a pillar of globalization, enabling economic development. It can facilitate the economic progress of a country, a region and local economy or a particular industry, such as tourism. A lack of air transport can hinder growth (Button, 2008). The aviation industry employs a large number of people and many more in support industries, such as hotels, restaurants, rental cars, real estate, construction and manufacturing. Many benefit economically from the air transport sector, without using their services, as a positive external effect (Wensveen, 2011).

This paper is focused on national airlines (also known as flag carriers, registered in a given state) in Europe - the EU and non-EU members. Flag carriers in the European Union operate in a highly competitive market, following liberalization in 1987-1997. As a consequence of the liberalization process every carrier having a license to offer air transport services to passengers, issued by any of the Member States, can fly on any route and offer any price of the service within the rules of free, fair and undisturbed competition (Button, 2001, Pisarek, 2009). National entities from non-EU countries, still benefiting from bilateral agreements, have to face the challenge of searching competitive advantage (Fitzgerald, 2011). Therefore, to establish the most efficient entities on the European passenger air transport market, DEA (Data Envelopment Analysis) methodology has been utilized. The DEA approach allows one to discriminate between homogeneous decision making units (or DMU's) - defined as the national passenger air carriers in Europe - based on their relative technical effectiveness. The aim of the research is to assess the effectiveness of 29 national air passenger carriers in Europe in the year 2013, by the DEA approach. Data were obtained from financial reports and statistics available on airline web sites, particularly in publications for investors. Calculations were performed in STATA software.

\section{Literature review}

European national airlines operate in a highly competitive environment and face the challenge of achieving effectiveness, in the struggle for higher revenue and profits. From the point of view of transport economics (Spurling, 2010), one can observe relatively high fixed costs in the airline industry and economies of scale. It is crucial, to rationalize fleet utilization and employment, to achieve the highest possible load factor of planes, by serving more passengers. Airlines benefit from the economy of scale (Button, 2010), economy of scope (Ben-Akiva, 
2008), economy of density (Vasigh, Tacker, Fleming, 2008) and capacity utilization (Jara-Diaz, Cortez, Moralez, 2013).

Since the measuring and improving of effectiveness in air transportation is an important issue, many researchers have incorporated the data envelopment approach. The DEA methodology is commonly used to assess airport efficiency. Adler and Berechman (2001) used the DEA approach to prove that West-European airports efficiency and quality are key factors airlines use in choosing hubs. Gutierrez and Lozano (2016) investigated the operational efficiency of 21 small and medium sized airports (SMAs) located in 10 different European countries. The results show that publicly owned SMAs have higher efficiencies than those that are privately owned and hub SMAs are more efficient than non-hub SMAs. Adler and Golany (2001) also proved the importance, or even super-efficiency, of hub airports by measuring the efficiency of some European airports under the effects of deregulation in air transportation.

Country-specific research has been carried out as well. An analysis of 21 Turkish airports suggested that the efficiency of most of them improved during the years 2009-2014, while operating hours and the percentage of international traffic were the main causes for inefficiency (Örkcü, Balıkç1, Dogan, Genç, 2016). In Nigeria an analysis of 30 airports’ efficiency (20032013) indicated that they have the ability to learn with their own managerial practices (Wanke, Barros, Nwaogbe, 2016). Fernandes and Pacheco (2002) performed a DEA analysis of 35 Brazilian domestic airports, comparing their passenger capacity to demand forecasts.

Gillen and Lall (1997; see also Gillen, Waters II, 1997) used DEA not for the operation of whole airports. They limited their research to terminals and airside operations. Their research shows that status as a hub airline and expanded gate capacity improves efficiency, while terminal efficiency grows with the number and utilization of gates. Liu (2016) decided to analyse the efficiency of airport management companies, instead of airports, in East Asia (10 companies in years 2009-2013). The results showed that Beijing Capital International Airport Co. Ltd. and Shanghai International Airport Co. Ltd. performed efficiently. Shao and Sun (2016) diverted their attention to the efficiency of air routes. They compared the efficiency of the transportation system, allocation, passenger traffic, and freight transport of 477 air routes concerning 82 airports in China. The results indicated that the majority of air routes have a high load factor and passenger transport efficiency, but relatively low cargo transport efficiency.

Researchers decided to use DEA to analyse the efficiency of airlines. Duygun, Prior, Shaban, and Tortosa-Ausina (2016) tried to assess the influence of recent deregulation and liberalization of air transportation in Europe. Their results show that customer satisfaction, cost minimization and efficient route systems are key factors for airlines. Chou, Lee, Chen, 
and Tsai (2016) researched the airline efficiency of 35 carriers divided into 2 regions, AsiaPacific and North America/Europe. The DEA approach has shown that airlines should put more focus on the reduction of input resources for productivity improvement. Generally, airlines in the Asia-Pacific area perform much better than those in N. America/Europe, due to technical efficiency and service effectiveness. Min and Joo (2016) used the research to measure airline alliances effectiveness. Airline strategic alliances are generally perceived to be a major driver in enhancing operating efficiency and subsequent competitiveness. However, the research did not prove that hypothesis. Most researchers combine passenger traffic and cargo transportation of all available airlines. In this paper, we have tried to extract only the efficiency of passenger transportation of European flag carriers.

\section{Data}

To measure the efficiency of national airlines 6 variables were chosen, three inputs (fleet, number of employees, number of destinations) and 3 outputs (total revenue, number of passengers, load factor). Data on inputs and outputs was gathered from corporate information, financial and annual reports, as well as statistics available on the websites of the mentioned carriers. Data was also collected from reports and statistics of airlines alliances, such as Star Alliance, One World and Sky Team. Taken into consideration were 29 national airlines and data available on the mentioned websites, is as follows:

1. From EU member countries: Adria Airways (Slovenia), Aegean Airlines (Greece), Aer Lingus (Ireland), Air Baltic (Latvia), Air Malta (Malta), Air Serbia (Serbia), Alitalia (Italy), Austrian Airlines (Austria), British Airways (Great Britain), Brussels Airlines (Belgium), Bulgaria Air(Bulgaria), Croatia Airlines (Croatia), Cyprus Airways (Cyprus), Czech Airlines (the Czech Republic), Estonian Air (Estonia), Finnair (Finland), Iberia Líneas Aéreas de España (Spain), KLM Royal Dutch Airlines (the Netherlands), LOT Polish Airlines (Poland), Lufthansa (Germany), Luxair (Luxembourg), SAS Scandinavian Airlines (Denmark, Norway, Sweden), TAP Portugal (Portugal), TAROM (Romania).

2. Non-EU European countries: Aeroflot (Russia), Icelandair (Iceland), SWISS (Switzerland), Turkish Airlines (Turkey), Ukraine International Airlines (Ukraine).

Generally, the statistical analysis indicates that national airlines in Europe are diverse. Some are small (with low inputs and low outputs): Cyprus Airways, Estonian Air, Air Malta, Adria Airways, and Bulgaria Air. Others generate very high outputs by using high inputs, such 
as: Lufthansa, Turkish Airlines, British Airways, KLM, and SWISS. This does not allow for passing judgment about the effectiveness of these airlines. However, the magnitude of each and all variables may be decisive while assessing effectiveness (as shown in Tables 1 and 2).

Table. 1 Statistical measures for inputs and outputs for 29 European national airlines in the year 2013

\begin{tabular}{|l|c|c|c|c|c|c|}
\cline { 2 - 8 } \multicolumn{1}{c|}{} & Fleet & Employees & Destinations & $\begin{array}{c}\text { Total revenue } \\
(\mathrm{mln} \text { USD })\end{array}$ & $\begin{array}{c}\text { Passangers } \\
(\mathrm{mln})\end{array}$ & $\begin{array}{c}\text { Load factor } \\
(\%)\end{array}$ \\
\hline Mean & 82.8 & $8,576.1$ & 86.3 & $4,090.9$ & 12.9 & 74.8 \\
\hline Median & 39 & 3,160 & 78 & 1,300 & 5.9 & 75.3 \\
\hline Min & 6 & 164 & 13 & 78 & 0.55 & 66 \\
\hline Max & 428 & 41,473 & 264 & 27,028 & 76.26 & 83.1 \\
\hline $\begin{array}{l}\text { Variation coeff. } \\
\text { (stand. deviation) (\%) }\end{array}$ & 119 & 136 & 75 & 159 & 131 & 6 \\
\hline
\end{tabular}

Source: own work.

\section{Fleet}

On average, each carrier possesses almost 83 aircraft; nearly half of the 29 airlines have no more than 39 planes. This combined with the variation coefficient (based on a standard deviation) of $119 \%$, suggest, that despite analysing only national carriers, there is a big dispersion between those companies. The least planes are owned by Cyprus Airways and the most by Lufthansa (as shown in Tables 1 and 2).

\section{Number of employees}

The mean number of employees equals 8,576 , while $50 \%$ of airlines have no more than 3,160 personnel. The variation is even higher then with the number of planes $-136 \%$ of the mean. The least employees are in Estonian Air, while the most are in British Airways $(41,475)$.

\section{Number of destinations (airports)}

The average number of airports served by each airline is 86.3 , while half of carriers fly to no more than 78 . The dispersion is $75 \%$. The smallest number of destinations is visited by Cyprus Airways and the most is in the Turkish Airlines (as show in Tables 1 and 2).

\section{Total revenue}

On the average, each airline generates a profit of 4,090.9 million USD a year, but half of carriers do not exceed 1,300 million USD. The standard deviation equals $159 \%$ of the mean. The smallest revenue was noted in Estonian Air and the highest profits in KLM (as show in Tables 1 and 2). 


\section{Passengers}

Every year each airline transports 12.9 million people, $50 \%$ of the analysed airlines take on-board 5.9 million people. The dispersion was $131 \%$. The least passengers are clients of Estonian Air and the most in Lufthansa (as show in Tables 1 and 2).

\section{Load factor}

The mean and median differ only slightly (mean 74.8\%, median 75.3\%), while the variation is very small (compared to other variables) $-6 \%$. The smallest load factor was in TAROM and the highest in KLM (as show in Tables 1 and 2).

Table. 2 Increasing order ranking of national carriers according to each of 6 variables in 2013

\begin{tabular}{|c|c|c|c|c|c|c|}
\hline 弟 & Fleet & Employees & Destinations & $\begin{array}{l}\text { Total revenue } \\
\text { (mln USD) }\end{array}$ & $\begin{array}{l}\text { Passengers } \\
\quad(\mathrm{mln})\end{array}$ & $\begin{array}{l}\text { Load factor } \\
\quad(\%)\end{array}$ \\
\hline 1 & 2 & 3 & 4 & 5 & 6 & 7 \\
\hline 1 & $\begin{array}{c}\text { Cyprus Airways } \\
\text { (6) }\end{array}$ & $\begin{array}{l}\text { Estonian Air } \\
\text { (164) }\end{array}$ & $\begin{array}{c}\text { Cyprus Airways } \\
\text { (13) }\end{array}$ & $\begin{array}{l}\text { Estonian Air } \\
\text { (78) }\end{array}$ & $\begin{array}{c}\text { Estonian Air } \\
(0.55)\end{array}$ & $\begin{array}{l}\text { TAROM } \\
(66)\end{array}$ \\
\hline 2 & $\begin{array}{l}\text { Estonian Air } \\
\text { (7) }\end{array}$ & $\begin{array}{c}\text { Adria Airways } \\
\quad(405)\end{array}$ & $\begin{array}{c}\text { Estonian Air } \\
\text { (17) }\end{array}$ & $\begin{array}{c}\text { Cyprus Airways } \\
(175.5)\end{array}$ & $\begin{array}{c}\text { Bulgaria Air } \\
(1.02)\end{array}$ & $\begin{array}{l}\text { Estonian Air } \\
\quad(66.3)\end{array}$ \\
\hline 3 & $\begin{array}{l}\text { Air Malta } \\
\text { (10) }\end{array}$ & $\begin{array}{c}\text { Czech Airlines } \\
\text { (925) }\end{array}$ & $\begin{array}{l}\text { Adria Airways } \\
\text { (18) }\end{array}$ & $\begin{array}{l}\text { Bulgaria Air } \\
\text { (181) }\end{array}$ & $\begin{array}{c}\text { Adria Airways } \\
(1.03)\end{array}$ & $\begin{array}{l}\text { Bulgaria Air } \\
(67.7)\end{array}$ \\
\hline 4 & $\begin{array}{c}\text { Adria Airways } \\
(10)\end{array}$ & $\begin{array}{c}\text { Croatia Airlines } \\
\text { (973) }\end{array}$ & $\begin{array}{l}\text { Bulgaria Air } \\
\text { (30) }\end{array}$ & $\begin{array}{c}\text { Adria Airways } \\
\text { (181) }\end{array}$ & $\begin{array}{c}\text { Air Serbia } \\
(1.06)\end{array}$ & $\begin{array}{c}\text { Croatia Airlines } \\
(69.1)\end{array}$ \\
\hline 5 & $\begin{array}{c}\text { Croatia Airlines } \\
\text { (12) }\end{array}$ & $\begin{array}{l}\text { Air Malta } \\
(1,011)\end{array}$ & $\begin{array}{c}\text { Croatia Airlines } \\
\text { (32) }\end{array}$ & $\begin{array}{l}\text { Air Malta } \\
(243)\end{array}$ & $\begin{array}{c}\text { Cyprus Airways } \\
(1.3)\end{array}$ & $\begin{array}{c}\text { Brussels Airlines } \\
(69.2)\end{array}$ \\
\hline 6 & $\begin{array}{l}\text { Bulgaria Air } \\
\text { (14) }\end{array}$ & $\begin{array}{c}\text { Air Baltic } \\
(1,100)\end{array}$ & $\begin{array}{l}\text { Air Malta } \\
(36)\end{array}$ & $\begin{array}{c}\text { Air Serbia } \\
(281)\end{array}$ & $\begin{array}{c}\text { Air Malta } \\
(1.43)\end{array}$ & $\begin{array}{c}\text { Czech Airlines } \\
(70.2)\end{array}$ \\
\hline 7 & $\begin{array}{l}\text { Luxair } \\
\text { (17) }\end{array}$ & $\begin{array}{c}\text { Cyprus Airways } \\
(1,226)\end{array}$ & $\begin{array}{c}\text { Icelandair } \\
\text { (39) }\end{array}$ & $\begin{array}{c}\text { Croatia Airlines } \\
(288)\end{array}$ & $\begin{array}{c}\text { Luxair } \\
(1.5)\end{array}$ & $\begin{array}{c}\text { Icelandair } \\
(70.7)\end{array}$ \\
\hline 8 & $\begin{array}{c}\text { Air Serbia } \\
\text { (19) }\end{array}$ & $\begin{array}{c}\text { Bulgaria Air } \\
(1,269)\end{array}$ & $\begin{array}{c}\text { Czech Airlines } \\
\text { (40) }\end{array}$ & $\begin{array}{l}\text { TAROM } \\
(322)\end{array}$ & $\begin{array}{c}\text { Croatia Airlines } \\
(1.8)\end{array}$ & $\begin{array}{c}\text { Cyprus Airways } \\
(71.1)\end{array}$ \\
\hline 9 & $\begin{array}{l}\text { TAROM } \\
(23)\end{array}$ & $\begin{array}{c}\text { Ukraine } \\
\text { International } \\
\text { Airlines } \\
(1,300)\end{array}$ & $\begin{array}{l}\text { TAROM } \\
(40)\end{array}$ & $\begin{array}{l}\text { Air Baltic } \\
\quad(351)\end{array}$ & $\begin{array}{c}\text { TAROM } \\
(2.2)\end{array}$ & $\begin{array}{l}\text { Air Baltic } \\
\text { (72) }\end{array}$ \\
\hline 10 & $\begin{array}{c}\text { Czech Airlines } \\
\text { (24) }\end{array}$ & $\begin{array}{l}\text { Aegean } \\
(1,357)\end{array}$ & $\begin{array}{l}\text { Air Serbia } \\
\quad(41)\end{array}$ & $\begin{array}{l}\text { Luxair } \\
(506)\end{array}$ & $\begin{array}{l}\text { Icelandair } \\
(2.26)\end{array}$ & $\begin{array}{c}\text { Ukraine } \\
\text { International } \\
\text { Airlines } \\
\text { (72) }\end{array}$ \\
\hline 11 & $\begin{array}{l}\text { Icelandair } \\
\text { (24) }\end{array}$ & $\begin{array}{c}\text { Air Serbia } \\
(1,527)\end{array}$ & $\begin{array}{l}\text { LOT Polish } \\
\text { Airlines } \\
(46)\end{array}$ & $\begin{array}{c}\text { Czech Airlines } \\
\text { (512) }\end{array}$ & $\begin{array}{c}\text { Czech Airlines } \\
(2.8)\end{array}$ & $\begin{array}{l}\text { Air Serbia } \\
\text { (73) }\end{array}$ \\
\hline 12 & $\begin{array}{l}\text { Air Baltic } \\
\text { (25) }\end{array}$ & $\begin{array}{l}\text { LOT Polish } \\
\text { Airlines } \\
(1,700)\end{array}$ & $\begin{array}{c}\text { Ukraine } \\
\text { International } \\
\text { Airlines } \\
(55)\end{array}$ & $\begin{array}{l}\text { Aegean } \\
(849)\end{array}$ & $\begin{array}{l}\text { Air Baltic } \\
(2.95)\end{array}$ & $\begin{array}{c}\text { Adria Airways } \\
\quad(73.2)\end{array}$ \\
\hline
\end{tabular}




\begin{tabular}{|c|c|c|c|c|c|c|}
\hline 1 & 2 & 3 & 4 & 5 & 6 & 7 \\
\hline 13 & $\begin{array}{l}\text { LOT Polish } \\
\text { Airlines } \\
(35)\end{array}$ & $\begin{array}{c}\text { TAROM } \\
(2,005)\end{array}$ & $\begin{array}{l}\text { Luxair } \\
\text { (59) }\end{array}$ & $\begin{array}{l}\text { LOT Polish } \\
\text { Airlines } \\
(1,010)\end{array}$ & $\begin{array}{c}\text { LOT Polish } \\
\text { Airlines } \\
(4.6)\end{array}$ & $\begin{array}{c}\text { Aegean } \\
(74.3)\end{array}$ \\
\hline 14 & $\begin{array}{l}\text { Aegean } \\
(36)\end{array}$ & $\begin{array}{l}\text { Luxair } \\
(2,288)\end{array}$ & $\begin{array}{l}\text { Air Baltic } \\
\quad(60)\end{array}$ & $\begin{array}{l}\text { Icelandair } \\
(1,022)\end{array}$ & $\begin{array}{c}\text { Ukraine } \\
\text { International } \\
\text { Airlines } \\
(4.6)\end{array}$ & $\begin{array}{c}\text { Alitalia } \\
(74.6)\end{array}$ \\
\hline 15 & $\begin{array}{c}\text { Ukraine } \\
\text { International } \\
\text { Airlines } \\
(39)\end{array}$ & $\begin{array}{l}\text { Icelandair } \\
(3,160)\end{array}$ & $\begin{array}{c}\text { Brussels Airlines } \\
\text { (78) }\end{array}$ & $\begin{array}{c}\text { Ukraine } \\
\text { International } \\
\text { Airlines } \\
(1,300)\end{array}$ & $\begin{array}{c}\text { Brussels Airlines } \\
\text { (5.9) }\end{array}$ & $\begin{array}{l}\text { Luxair } \\
(75.3)\end{array}$ \\
\hline 16 & $\begin{array}{c}\text { Brussels Airlines } \\
(45)\end{array}$ & $\begin{array}{c}\text { Brussels Airlines } \\
\qquad(3,500)\end{array}$ & $\begin{array}{l}\text { Aer Lingus } \\
\quad(80)\end{array}$ & $\begin{array}{c}\text { Brussels Airlines } \\
(1,440)\end{array}$ & $\begin{array}{l}\text { Aegean } \\
(6.9)\end{array}$ & $\begin{array}{c}\text { SAS } \\
\text { Scandinavian } \\
\text { Airlines } \\
(76.6)\end{array}$ \\
\hline 17 & $\begin{array}{c}\text { Aer Lingus } \\
(47)\end{array}$ & $\begin{array}{c}\text { Aer Lingus } \\
(4,000)\end{array}$ & $\begin{array}{c}\text { Alitalia } \\
(83)\end{array}$ & $\begin{array}{c}\text { Aer Lingus } \\
(1,532)\end{array}$ & $\begin{array}{c}\text { Finnair } \\
(9.6)\end{array}$ & $\begin{array}{c}\text { TAP Portugal } \\
(76.9)\end{array}$ \\
\hline 18 & $\begin{array}{l}\text { Finnair } \\
(67)\end{array}$ & $\begin{array}{l}\text { Finnair } \\
(5,859)\end{array}$ & $\begin{array}{l}\text { SWISS } \\
(84)\end{array}$ & $\begin{array}{c}\text { Austrian Airlines } \\
(2,069)\end{array}$ & $\begin{array}{l}\text { Aer Lingus } \\
\quad(9.6)\end{array}$ & $\begin{array}{l}\text { LOT Polish } \\
\text { Airlines } \\
(77)\end{array}$ \\
\hline 19 & $\begin{array}{c}\text { TAP Portugal } \\
(77)\end{array}$ & $\begin{array}{c}\text { Austrian Airlines } \\
(6,108)\end{array}$ & $\begin{array}{c}\text { TAP Portugal } \\
(88)\end{array}$ & $\begin{array}{l}\text { Finnair } \\
(2,627)\end{array}$ & $\begin{array}{c}\text { TAP Portugal } \\
(10.7)\end{array}$ & $\begin{array}{c}\text { Air Malta } \\
(77.2)\end{array}$ \\
\hline 20 & $\begin{array}{c}\text { Austrian Airlines } \\
(80)\end{array}$ & $\begin{array}{c}\text { TAP Portugal } \\
(6,889)\end{array}$ & $\begin{array}{l}\text { Iberia } \\
(100)\end{array}$ & $\begin{array}{c}\text { TAP Portugal } \\
(3,070)\end{array}$ & $\begin{array}{c}\text { Austrian Airlines } \\
(11.3)\end{array}$ & $\begin{array}{c}\text { Turkish Airlines } \\
\text { (77.4) }\end{array}$ \\
\hline 21 & $\begin{array}{l}\text { SWISS } \\
(90)\end{array}$ & $\begin{array}{l}\text { SWISS } \\
(8,250)\end{array}$ & $\begin{array}{c}\text { Finnair } \\
(106)\end{array}$ & $\begin{array}{l}\text { Alitalia } \\
(3,325) \\
\end{array}$ & $\begin{array}{l}\text { SWISS } \\
(15.97)\end{array}$ & $\begin{array}{c}\text { Aer Lingus } \\
(78.4)\end{array}$ \\
\hline 22 & $\begin{array}{c}\text { Alitalia } \\
(118)\end{array}$ & $\begin{array}{c}\text { Alitalia } \\
(11,726)\end{array}$ & $\begin{array}{c}\text { Aegean } \\
(120)\end{array}$ & $\begin{array}{c}\text { Iberia } \\
(4,578)\end{array}$ & $\begin{array}{l}\text { Iberia } \\
(20.8)\end{array}$ & $\begin{array}{c}\text { Austrian Airlines } \\
(78.6)\end{array}$ \\
\hline 23 & $\begin{array}{r}\text { Iberia } \\
(128)\end{array}$ & $\begin{array}{c}\text { SAS } \\
\text { Scandinavian } \\
\text { Airlines } \\
(12,548) \\
\end{array}$ & $\begin{array}{c}\text { SAS } \\
\text { Scandinavian } \\
\text { Airlines } \\
(123)\end{array}$ & $\begin{array}{l}\text { SWISS } \\
(5,170)\end{array}$ & $\begin{array}{l}\text { Aeroflot Russian } \\
\text { Airlines } \\
(20.9)\end{array}$ & $\begin{array}{l}\text { Iberia } \\
(78.6)\end{array}$ \\
\hline 24 & $\begin{array}{c}\text { SAS } \\
\text { Scandinavian } \\
\text { Airlines } \\
(142)\end{array}$ & $\begin{array}{c}\text { Aeroflot Russian } \\
\text { Airlines } \\
(17,800)\end{array}$ & $\begin{array}{c}\text { Austrian Airlines } \\
(130)\end{array}$ & $\begin{array}{c}\text { SAS } \\
\text { Scandinavian } \\
\text { Airlines } \\
(5,940)\end{array}$ & $\begin{array}{c}\text { Alitalia } \\
(23.2)\end{array}$ & $\begin{array}{l}\text { Lufthansa } \\
\text { (78.8) }\end{array}$ \\
\hline 25 & \begin{tabular}{|c|} 
Aeroflot Russian \\
Airlines \\
$(158)$ \\
\end{tabular} & $\begin{array}{c}\text { Iberia } \\
(18,000)\end{array}$ & $\begin{array}{c}\text { Aeroflot Russian } \\
\text { Airlines } \\
(131)\end{array}$ & $\begin{array}{c}\text { Aeroflot Russian } \\
\text { Airlines } \\
(9,140)\end{array}$ & $\begin{array}{l}\text { KLM } \\
(26.6)\end{array}$ & $\begin{array}{c}\text { Aeroflot Russian } \\
\text { Airlines } \\
(78.8)\end{array}$ \\
\hline 26 & $\begin{array}{l}\text { KLM } \\
(201)\end{array}$ & $\begin{array}{c}\text { Turkish Airlines } \\
(19,658)\end{array}$ & $\begin{array}{l}\text { KLM } \\
(144)\end{array}$ & $\begin{array}{c}\text { Turkish Airlines } \\
(9,560)\end{array}$ & $\begin{array}{c}\text { SAS } \\
\text { Scandinavian } \\
\text { Airlines } \\
(27.1)\end{array}$ & $\begin{array}{l}\text { Finnair } \\
(79.5)\end{array}$ \\
\hline 27 & $\begin{array}{c}\text { British Airways } \\
(258)\end{array}$ & $\begin{array}{c}\text { KLM } \\
(32,505)\end{array}$ & $\begin{array}{c}\text { British Airways } \\
(212)\end{array}$ & $\begin{array}{c}\text { British Airways } \\
(17,304)\end{array}$ & $\begin{array}{c}\text { British Airways } \\
(33.8)\end{array}$ & $\begin{array}{c}\text { British Airways } \\
(81)\end{array}$ \\
\hline 28 & $\begin{array}{c}\text { Turkish Airlines } \\
(260) \\
\end{array}$ & $\begin{array}{c}\text { Lufthansa } \\
(39,981)\end{array}$ & $\begin{array}{c}\text { Lufthansa } \\
(235)\end{array}$ & $\begin{array}{c}\text { Lufthansa } \\
(18,555)\end{array}$ & $\begin{array}{c}\text { Turkish Airlines } \\
(46.16)\end{array}$ & $\begin{array}{l}\text { SWISS } \\
(82.6)\end{array}$ \\
\hline 29 & $\begin{array}{c}\text { Lufthansa } \\
(428)\end{array}$ & $\begin{array}{c}\text { British Airways } \\
(41,473)\end{array}$ & $\begin{array}{c}\text { Turkish Airlines } \\
\text { (264) }\end{array}$ & $\begin{array}{c}\text { KLM } \\
(27,028)\end{array}$ & $\begin{array}{c}\text { Lufthansa } \\
(76.26)\end{array}$ & $\begin{array}{l}\text { KLM } \\
(83.1) \\
\end{array}$ \\
\hline
\end{tabular}

Source: own work (values of variables are given in brackets). 


\section{Method}

Data Envelopment Analysis (DEA) is a multidimensional nonparametric optimization approach that has become a popular analytical tool for the quantitative valuation of efficiency of organizations, in both the private and public sector. The basic concept of DEA assumes the existence of a production possibility frontier or efficiency frontier and calculates the quantitative distance between the input position of a given DMU (decision making unit) and the frontier (for input oriented approach) or output position to the frontier (for output oriented models), (Kourtit, Nijkamp, 2013; Toloo, Nalchigar, 2009). The efficiency is relative, as it is based on a comparison between DMU, chosen for the analysis. Each object is described by multiple inputs and outputs (Suzuk, Nijkamp, 2011; Appa, Williams, 2002; Lee, Worthington, 2016). The combination of inputs and outputs of all DMUs allows for drawing a multidimensional effectiveness (efficiency) frontier, based on objects that are fully effective with their linear combinations (Farrell, 1957). For every DMU an efficiency coefficient $\theta$ is calculated, if its value is (Suzuki, Nijkamp, Rietveld, 2011):

- equal to $100 \%-100 \%$ of the resources are fully used and transformed into outputs DMU is efficient and on the frontier,

- less than $100 \%$ (for input oriented models) or larger than $100 \%$ (for output oriented models) - DMUs are inefficient and part of the inputs are being wasted and/or part of the outputs which could be produced, is not.

Results of the DEA approach indicate which changes (ceteris paribus) should be employed to achieve the full 'production' potential. If the DEA analysis relative to technical efficiency for $N$ objects (DMUs), based on $M$ inputs and $S$ outputs, where: $D M U_{k}-k^{\text {th }}$ Decision Making Unit (DMU), $k=1, \ldots, N, Y_{r k}-r^{\text {th }}$ output of $k^{\text {th }}$ DMU, $r=1, \ldots, S, X_{i k}-i^{\text {th }}$ input of $k^{\text {th }}$ DMU, $i=1, \ldots, M$, then for each DMU the optimization is:

$$
\begin{gathered}
\max _{\mu, \vartheta} \frac{\sum_{1}^{S} \mu_{r k} \times y_{r k}}{\sum_{1}^{M} \vartheta_{i k} \times x_{i k}} \\
\frac{\sum_{1}^{S} \mu_{r k} \times y_{r j}}{\sum_{1}^{M} \vartheta_{i k} \times x_{i j}} \leq 1 \\
\mu_{r k} \geq 0, \vartheta_{i k} \geq 0 \\
j, k=1, \ldots, N ; \quad r=1, \ldots, S ; \quad i=1, \ldots, M
\end{gathered}
$$

$\mu_{r k}, \vartheta_{i k}-$ parameters (weights, multipliers) calculated to maximize the efficiency. 
As a result for each DMU the efficiency coefficient $\theta$ and vectors of slacks $\left(\mathrm{s}^{-}\right)$and surpluses $\left(\mathrm{s}^{+}\right)$are calculated. Therefore, the point on the frontier closeted to inefficient DMU (or its image on the frontier), is defined as:

for input oriented models (minimization of inputs)

$$
\left(\theta \times x_{k}-s^{-} ; \quad y_{k}+s^{+}\right)
$$

for output oriented models (maximization of outputs)

$$
\left(x_{k}-s^{-} ; \quad \theta \times y_{k}+s^{+}\right)
$$

(for more see Charnes, Cooper, Rhodes, 1978; Suzuk, Nijkamp, 2011; Domagała, 2007).

Moving or projecting an inefficient DMU toward the frontier by the transformation of 5 or 6 (depending on model orientation) using the efficiency coefficient $\theta$ and vectors of slacks and surpluses should allow for achieving the efficiency frontier (Kourtit, Nijkamp, 2013; Toloo, Nalchigar, 2009; Sherman, Zhu, 2006).

The idea of the classic DEA approach is the recipe for an instantaneous improvement, hidden in formulas 5 and 6 . However, in some cases the calculated change needed for 'moving' an object to the frontier is very drastic, for example reducing all inputs by $50 \%$. This is rarely possible in real conditions, for any decision making. Therefore, some modifications of the basic DEA model have been introduced. CD DEA is one of those alternations. Context-Dependent (Stepwise improvement) DEA assumes, that a small improvement is better than none. It divides the set of DMUs not only into two subsets: efficient (laying on the efficiency frontier) and inefficiency (off the frontier). It draws several frontiers, each constructed on different objects. The first frontier is the same one, as in the classic approach, based on the fully effective DMUs. The second (below the first), ignores objects on the first level and uses DMUs with slightly inferior technical effectiveness. The third incorporates even worse objects and so on. In this approach the goal is to improve inefficient DMUs, by moving them to an upper level, not necessary the top level, with full efficiency (Seiford, Zhu, 2003; Suzuk, Nijkamp, 2011). In this paper both, the classic and Context-Dependent, have been used.

\section{Results}

To analyse the relative technical effectiveness (or efficiency) of national carriers (29 DMUs) in Europe, 2 models were employed: (classic) DEA and CD DEA (Context-Dependent, Stepwise improvement DEA). Both models are input oriented, as inputs are easier to modify by decision makers than the outputs. ${ }^{1}$

\footnotetext{
1 Both models assume constant effects of scale. Calculations were made in STATA MP 11.
} 
The classic DEA approach suggested that out of 29 airlines, 12 are fully efficient, such as: Cyprus Airways, SAS Scandinavian Airlines, Estonian Air, Aegean, KLM, Aer Lingus, Lufthansa, Adria Airways, Alitalia, SWISS, Turkish Airlines, and Ukraine International Airlines. The remaining 17 "waste" some of their inputs, and in some cases underachieve their outcomes. Using formula 5, for instance, for LOT Polish Airlines, on original input and output values (see Table 2) and DEA results (see Table 3), the projected point on the efficiency frontier may be calculated, as follows:

$$
\begin{gathered}
\left(\theta \times x_{k}-s^{-} ; y_{k}+s^{+}\right)=\left(98 \% \times\left[\begin{array}{lll}
35 & 1,700 & 46
\end{array}\right]-\left[\begin{array}{lll}
0 & 0 & 0
\end{array}\right] ;\left[\begin{array}{lll}
1,010 & 4.6 & 77
\end{array}\right]+\left[\begin{array}{lll}
129.5 & 0 & 0
\end{array}\right]\right)= \\
=\left(\left[\begin{array}{lll}
34.3 & 1,666 & 45.1
\end{array}\right] ;\left[\begin{array}{lll}
1,139.5 & 4.6 & 77
\end{array}\right]\right)
\end{gathered}
$$

Among the inefficient carriers, there are some with quite high efficiency coefficients (above 90\%), like LOT, which uses 98\% of its resources and Czech Airlines, Air Malta, and Croatia Airlines. To achieve full efficiency, they would have to slightly decrease all their inputs (proportionally by less than 10\%) and, in the case of Polish and Czech Airlines, increase their revenue, by almost \$ 130 million and \$ 137 million, respectively, while Air Malta should additionally decrease the number of destinations by 11 (after the proportional reduction of inputs). On the other hand, some airlines overestimated their inputs by over $40 \%$ and therefore their efficiency level was lower than 60\%: Air Serbia $(\theta=46 \%)$, Bulgaria Air $(\theta=54 \%)$, $\operatorname{TAROM}(\theta=56 \%)$, and Luxair $(\theta=57 \%)$. For these carriers, gaining full efficiency in 2013 was practically impossible, as cutting all inputs (number of aircraft, employees, and destinations) by half, would cause social issues for example with unions, market (by selling numerous planes), and regional authorities. A major reduction in employment, would undoubtedly influence at least some local labour markets, while reducing the possible number of destinations, would decrease some regions business and touristic attractiveness. Overgrown employment was the biggest problem for British Airways and Iberia, that beside the proportional reduction of all inputs connected with the efficiency coefficient, required an additional adjustment $(15,800$ and 6,800, respectfully). For many destinations, served by Air Malta and Luxair, the total reduction, needed for full efficiency, was almost 14 out of 36 and 41 out of 59, respectively. No additional adjustment was necessary for fleets of inefficient airlines. Additional increments could be expected for Polish Airlines, Czech Airlines, Air Baltic, and TAROM, for the total revenue by almost \$130, \$137, \$108, and \$ 24 million, respectively. The load factor was an issue only for British Airways. Its value of $81 \%$ was too low and although it would not be possible to increase it by 61.6 p.p. reaching more than $100 \%$, some improvement in this aspect of outputs should be 
considered. The annual number of passengers served, was satisfactory for all carriers (as shown in Table 3).

In all inefficient Using Context Dependent DEA models, there are 4 levels of efficiency frontiers, among 29 national airlines in Europe. The first level, with fully efficient carriers, is the same as in the classic DEA. The second encompasses 10 airlines, some with very high results of the classic DEA - LOT Polish Airlines, Czech Airlines, Air Malta, and Croatia Airlines - but also much less effective ones - Icelandair or Bulgaria Air.

Table. 3 Necessary corrections for inputs (efficiency coefficient, proportional reduction, slacks) and outputs (surpluses) of inefficient airlines for achieving full efficiency in the year 2013

\begin{tabular}{|c|c|c|c|c|c|c|c|c|}
\hline \multirow[b]{3}{*}{ Inefficient airlines } & \multirow[b]{3}{*}{$\begin{array}{c}\text { Efficiency } \\
\text { coefficient } \\
\theta \\
(\%)\end{array}$} & \multicolumn{4}{|c|}{ Inputs reduction } & \multirow{2}{*}{\multicolumn{3}{|c|}{$\begin{array}{c}\text { Outputs increase } \\
\text { Additional (surpluses s }{ }^{+} \text {) }\end{array}$}} \\
\hline & & \multirow[b]{2}{*}{$\begin{array}{c}\text { Proportional } \\
(100 \%-\theta) \\
(\%)\end{array}$} & \multicolumn{3}{|c|}{ Additional (slacks s-) } & & & \\
\hline & & & 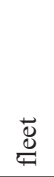 & $\begin{array}{l}\tilde{d} \\
\stackrel{0}{0} \\
\text { 范 }\end{array}$ & 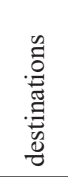 & 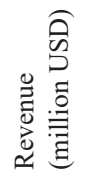 & 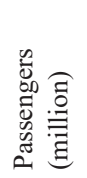 & 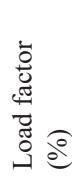 \\
\hline LOT Polish Airlines & 98 & 2 & & & & 129.5 & & \\
\hline Czech Airlines & 93 & 7 & & & & 136.6 & & \\
\hline Air Malta & 92 & 8 & & & 10.7 & & & \\
\hline Croatia Airlines & 91 & 9 & & & & & & \\
\hline Iberia & 86 & 14 & & $4,273.9$ & & & & \\
\hline British Airways & 80 & 20 & & $7,500.6$ & & & & 61.5 \\
\hline $\begin{array}{l}\text { Aeroflot Russian } \\
\text { Airlines }\end{array}$ & 78 & 22 & & & & & & \\
\hline Finnair & 78 & 22 & & & & & & \\
\hline TAP Portugal & 77 & 23 & & & & & & \\
\hline Air Baltic & 76 & 24 & & & & 107.5 & & \\
\hline Austrian Airlines & 73 & 27 & & & & & & \\
\hline Brussels Airlines & 73 & 27 & & & & & & \\
\hline Icelandair & 60 & 40 & & & & & & \\
\hline Luxair & 57 & 43 & & & 15.3 & & & \\
\hline TAROM & 56 & 44 & & & & 24.2 & & \\
\hline Bulgaria Air & 54 & 46 & & & & & & \\
\hline Air Serbia & 46 & 54 & & & & & & \\
\hline
\end{tabular}

Source: own work based on STATA optimization results (add your calculation to the appendix).

The efficiency coefficients are very dispersed on this frontier (53.6-97.8\%). The third level frontier encloses 6 carriers, including 3 of the worst DMUs of classic DEA - Air Serbia, TAROM, and Luxair. Being on the $3^{\text {rd }}$ frontier, they are relatively close to the upper $2^{\text {nd }}$ level, as effectiveness coefficients vary only from 80.3 to $99.7 \%$. On the last level there is only 
one carrier - Brussels Airlines, very near the upper $3^{\text {rd }}$ frontier, as it is lacking only $1.2 \%$ of effectiveness. Surprisingly, this airline was not among the bottom five carriers, in the classic DEA results. In most cases, the improvement to the nearest upper level frontier is smaller and easier to implement then the overall change, suggested in the primary approach (as shown in Table 4).

Table. 4 Four efficiency frontiers of European national air carriers in the year 2013

\begin{tabular}{|c|c|}
\hline $\begin{array}{c}\text { Frontier } \\
\text { level }\end{array}$ & Number of airlines on frontier: Airline (Efficiency coefficient to upper frontier) \\
\hline 1 & $\begin{array}{l}\text { 12: Cyprus Airways, SAS Scandinavian Airlines, Estonian Air, Aegean, KLM, Aer Lingus, Lufthansa, } \\
\text { Adria Airways, Alitalia, SWISS, Turkish Airlines, Ukraine International Airlines }(100 \%)\end{array}$ \\
\hline 2 & $\begin{array}{l}\text { 10: LOT Polish Airlines (97.8\%), Czech Airlines (92.9\%), Air Malta (91.7\%), Croatia Airlines (90.5\%), } \\
\text { Iberia (85.7\%), British Airways (79.7\%), Aeroflot Russian Airlines (78.1\%), Finnair (78.0\%), Icelandair } \\
(60.5 \%) \text {, Bulgaria Air (53.7\%) }\end{array}$ \\
\hline 3 & $\begin{array}{l}\text { 6: TAP Portugal (99.7\%), Austrian Airlines (98.7\%), Air Baltic (96.4\%), Air Serbia (81.4\%), TAROM } \\
(80.5 \%) \text {, Luxair (80.3\%) }\end{array}$ \\
\hline 4 & 1: Brussels Airlines $(98.8 \%)$ \\
\hline
\end{tabular}

Source: own work based on STATA optimizations results.

\section{Conclusions}

Air transportation and passenger flag carriers influence economic development. They employ vasts numbers of people, which has a direct connection to labour market and indirect income effects on the economy. Moreover, evadible destinations, their number and directions, will have an impact on stimulating or hindering the attractiveness of regions for business and tourism. Political and economic circumstances will influence demand for travelling, associated services, both for business and leisure purposes. Some events, like an economic crisis, war, terrorism, epidemics, or embargoes will affect the demand side, but also the procedures and functioning of airlines.

The DEA analysis shows, that more than 40\% (12 of 29) of the researched airlines, are effective and another 34\% (10 of 29) are near-efficient. Moreover, results suggest that "going big" may not increase effectiveness. It is harder to achieve full efficiency for big carriers, than small ones. This stands in opposition to regional policies and the interests of increasing employment, as well as tourism and business attractiveness. In the end, national airlines are companies existing on a free market, with limited resources. If the inefficient airlines decide to improve, they will most likely be forced to reduce their inputs. This course has been confirmed by both the classic DEA and CD DEA approach. 


\section{References}

Adler, N., Berechman, J. (2001). Measuring airport quality from the airlines' viewpoint: an application of data envelopment analysis. Transport policy, 8, 171-181.

Adler, N., Golany, B. (2001). Evaluation of deregulated airline networks using data envelopment analysis combined with principal component analysis with an application to Western Europe. European Journal of Operational Research, 132, 260-273.

Andersen M.M., Poulfelt, F. (2006). Discount Business Strategy. How the New Market Leaders are redefining Business Strategy. Chichester Southern Gate: John Wiley \& Sons Ltd.

Appa, G., Williams, H.P. (2002). A formula for the solution of DEA models. Operational Research working paper, LSEOR 02.49. London: Department of Operational Research, London School of Economics and Political Science.

Ben-Akiva, M. (2008). Transportation Costs. Transportation Systems Analysis: Demand \& Economics. Cambridge: Massachusetts Institute of Technology.

Button, K. (2001). Deregulation and Liberalization of European Air Transport Markets. Innovation. The European Journal of Social Sciences, 14 (3).

Button, K. (2008). The impact of globalization on international air transport activity. Past trends and future perspectives. OECD: International Transport Forum.

Button, K. (2010). Transport economics, $3^{\text {rd }}$ Edition. Edward Elgar Publishing.

Charnes, A., Cooper, W., Rhodes, A. (1978). Measuring the efficiency of decision making units. European Journal of Operations Research, 2, 429-444.

Chou, H.-W., Lee, C.-Y., Chen, H.-K., Tsai, M.-Y. (2016). Evaluating airlines with slack-based measures and meta-frontiers. Journal of Advanced Transportation, 50, 1061-1089. DOI: 10.1002/atr.1390.

Domagała, A. (2007). Metoda data envelopment analysis jako narzędzie badania względnej efektywności technicznej. Badania Operacyjne i Decyzje, 3-4, 22-34.

Duygun, M., Prior, D., Shaban, M., Tortosa-Ausina, E. (2016). Disentangling the European airlines efficiency puzzle: A network data envelopment analysis approach. Omega, 60, 2-14.

Farrell, M.J. (1957). Measurement of Productive Efficiency. Journal of Royal Statistical Society.

Fernandes, E., Pacheco, R.R. (2002). Efficient use of airport capacity. Transportation Research Part A, 39, 228-238.

Fitzgerald, P. (2011). In defense of the nationality of aircraft. Annals of Air and Space Law, 87.

Gillen, D., Lall, A. (1997). Developing measures of airport productivity and performance: an application of data envelopment analysis. Transportation Research Part E: Logistics and Transportation Review, 33 (4), 261-273. 
Gillen, D., Waters II, W.G. (1997). Introduction. Airport performance measurement and airport pricing. Transportation Research Part E: Logistics and Transportation Review, 33 (4), 245-247.

Gutierrez, E., Lozano, S. (2016). Efficiency assessment and output maximization possibilities of European small and medium sized airports. Research in Transportation Economics, 56, 3-14.

Jara-Diaz, S., Cortez, C., Moralez, G. (2013). Explaining changes and trends in the airline industry: Economies of density, multiproduct scale, and spatial scope. Transportation Research, 60, 13-26.

Konert, A. (2014). A European Vision for Air Passengers. Warsaw: Lazarski University Publishing House.

Kourtit, K., Nijkamp, P. (2013). In search of creative champions in high-tech spaces: a spatial application of strategic performance management. Journal of regional science, 53 (5), 749-777.

Lee, B.L., Worthington, A.C. (2016). A network DEA quantity and quality-orientated production model: An application to Australian university research services. Omega, 60, 26-33.

Liu, D. (2016). Measuring aeronautical service efficiency and commercial service efficiency of East Asia airport companies: An application of Network Data Envelopment Analysis. Journal of Air Transport Management, 52, 11-22.

Min, H., Joo, J.-S. (2016). A comparative performance analysis of airline strategic alliances using data envelopment analysis. Journal of Air Transport Management, 52, 99-110.

Örkcü, H.H., Balıcçı, C., Dogan, M.I., Genç, A. (2016). An evaluation of the operational efficiency of Turkish airports using data envelopment analysis and the Malmquist productivity index: 2009-2014 case. Transport Policy, 48, 92-104.

Pisarek, R. (2009). Air transport liberalization in the European Union and its impact on development of the civil aviation sector in Poland. Comparative Economic Research Central and Eastern Europe, 12 (2), 45-58.

Reichmuth, J. (2008). Analyses of the European Air Transport Market. Airline Business Models. Köln: German Aerospace Center.

Seiford, L.M., Zhu, J. (2003). Context-dependent Data Envelopment Analysis - Measuring attractiveness and progress. Omega, 31, 397-408.

Shao, Y., Sun, C. (2016). Performance evaluation of China's air routes based on network data envelopment analysis approach. Journal of Air Transport Management, 55, 67-75.

Sherman, H.D., Zhu J. (2006). Service Productivity Management: Improving Service Performance using Data Envelopment Analysis (DEA). Springer Science \& Business Media.

Spurling, D.J. (2010). Introduction to Transport Economics. Demand, Cost, Pricing, and Adoption. Boca Raton: Universal Publishing. 
Suzuk, S., Nijkamp, P. (2011). A Stepwise Projection Data Envelopment Analysis for Public Transport Operations in Japan. Tinbergen Institute Discussion Paper, TI 2011-113/3.

Suzuki, S., Nijkamp, P., Rietveld, P. (2011). Regional Efficiency Improvement by Means of Data Envelopment Analysis through Euclidean Distance Minimization including Fixed Input Factors - An Application to Tourist Regions in Italy. Tinbergen Institute Discussion Paper. TI 2011-110/3.

Toloo, M., Nalchigar, S. (2009). A new integrated DEA model for finding most BCC-efficient DMU. Applied Mathematical Modelling, 33, 597-604.

Vasigh, B., Tacker, T., Fleming, K. (2008). Introduction to Air Transport Economics: From Theory to Applications. Aldershot: Ashgate.

Wanke, P., Barros, C.P., Nwaogbe, O.R. (2016). Assessing productive efficiency in Nigerian airports using Fuzzy-DEA. Transport Policy, 49, 9-19.

Wensveen J.G. (2011). Air Transportation. A Management Perspective. Burlington: Ashgate Publishing.

Financial reports, statistics and data available on web sites of European flag airlines:

Adria Airways, Slovenia (www.adria.si).

Aegean Airlines, Greece (www.en.aegeanair.com).

Aer Lingus, Ireland (www.aerlingus.com).

Aeroflot Russian Airlines, Russia (www.aeroflot.com).

Air Baltic, Latvia (www.airbaltic.com).

Air Malta, Malta (www.airmalta.com).

Air Serbia, Serbia (www.airserbia.com).

Alitalia, Italy (www.alitalia.com).

Austrian Airlines, Austria (www.austrian.com).

British Airways, Great Britain (www.britishairways.com).

Brussels Airlines, Belgium (www.brusselsairlines.com).

Bulgaria Air, Bulgaria (www.air.bg).

Croatia Airlines, Croatia (www.croatiaairlines.com).

Cyprus Airways, Cyprus (www.cyprusair.com).

Czech Airlines, the Czech Republic (www.czechairlines.com).

Estonian Air, Estonia (www.estonian-air.ee).

Finnair, Finland (www.finnair.com).

Iberia Líneas Aéreas de España, Spain (www.iberia.com).

Icelandair, Iceland (www.icelandair.com).

KLM Royal Dutch Airlines, the Netherlands (www.klm.com). 
LOT Polish Airlines, Poland (www.lot.com).

Lufthansa, Germany (www.lufthansa.com).

Luxair, Luxembourg (www.luxair.lu).

SAS Scandinavian Airlines, Denmark, Norway, Sweden (www.sasgroup.net).

SWISS, Switzerland (www.swiss.com).

TAP Portugal, Portugal (www.tapportugal.com).

TAROM, Romania (www.tarom.ro).

Turkish Airlines, Turkey (www.turkishairlines.com).

Ukraine International Airlines, Ukraine (www.flyuia.com). 